\title{
A unique array of neuroprotective effects of pyruvate in neuropathology
}

\author{
Yuri Zilberter *, Olena Gubkina and Anton I. Ivanov \\ ${ }^{*}$ Correspondence: yuri.zilberter@univ-amu.fr \\ Edited by: \\ Pierre J. Magistretti, École Polytechnique Fédérale de Lausanne, Switzerland \\ Reviewed by: \\ Avital Schurr, University of Louisville, USA \\ Evelyne Gozal, University of Louisville, USA
}

Institut de Neurosciences des Systèmes, Aix Marseille Université, Inserm UMR_S 1106, Marseille, France

Keywords: energy metabolism, pyruvate, neurodegeneration, oxidative stress, PARP-1, NAD, neuroinflammation

The three common signature characteristics of many neurological diseases are brain hypometabolism, oxidative stress, and neuroinflammation (Melo et al., 2011; Cai et al., 2012; Heneka et al., 2014). In order to be efficient, successful treatment should target all three pathologies simultaneously. Pyruvate seems to be an ideal candidate for such a treatment because of its unique combination of neuroprotective effects (Figure 1). In this opinion paper, we attempt to review and summarize recent information concerning these effects and their significance for neuroprotection.

\section{PYRUVATE ENHANCES THE} \section{BRAIN-TO-BLOOD GLUTAMATE EFFLUX}

Perisynaptic astrocytes normally provide fast take-up of glutamate released during synaptic activity. In pathological conditions however, extracellular glutamate levels can be abnormally high and neurotoxic (Wang and Qin, 2010). Part of this glutamate can be cleared via glutamate transporters located in the capillary endothelial cells that form the bloodbrain-barrier. The efficacy of such efflux depends on the glutamate concentration gradient between blood and interstitial fluid (Teichberg et al., 2009). Meanwhile, blood glutamate content can be lowered by activation of a blood-resident enzyme glutamate-pyruvate transaminase that in the presence of pyruvate transforms glutamate into 2-ketoglutarate (Gottlieb et al., 2003), thus reducing the glutamate blood concentration. This should favor the glutamate flux from the interstitial fluid to the blood. Therefore, extracellular glutamate levels can be controlled in part by blood pyruvate, which can enhance the brain-to-blood glutamate efflux.

Indeed, Zlotnik and co-authors demonstrated (Zlotnik et al., 2008, 2012) that intravenous injection of pyruvate after traumatic brain injury in rats led to a transient decrease in blood glutamate levels and significantly improved neurological outcome during the first days following injury as well as hippocampal neuron survival at 30 days after injury.

One of the most severe acute neurological conditions, associated with excessive glutamate release, is the status epilepticus (SE). Glutamate-induced excitotoxicity is largely based on massive influx of $\mathrm{Ca}^{2+}$ via glutamate receptors, which seems to be a necessary step in the overall process of neuronal degeneration and the acute neuronal cell death that occurs after SE. Morphological analysis of the rat brain after pilocarpine-induced SE demonstrates that the hippocampal subfield CA1 and the hilus of dentate gyrus are particularly susceptible to neuronal cell loss. SEinduced neuronal loss in CA1 was largely prevented in rats treated with pyruvate plus oxaloacetate (i.p. injection $30 \mathrm{~min}$ after development of SE) (Carvalho et al., 2011). Moreover, neuronal damage in the dentate gyrus was prevented in rats that received pyruvate alone while oxaloacetate alone did not reveal any neuroprotective effects. The authors related the observed beneficial effects to the blood glutamate scavenging, although other capabilities of pyruvate could also influence the positive outcome.

\section{PYRUVATE NON-ENZYMATICALLY SCAVENGES $\mathrm{H}_{2} \mathrm{O}_{2}$}

Pyruvate in relatively small concentrations $(<1 \mathrm{mM})$ protects neurons against $\mathrm{H}_{2} \mathrm{O}_{2}$-induced toxicity (Desagher et al., 1997). This effect is not related to the pyruvate's function as an energy substrate but rather to its ability to non-enzymatically interact with $\mathrm{H}_{2} \mathrm{O}_{2}$ producing acetate, water, and carbon dioxide (Holleman, 1904). The antioxidant effects of pyruvate and other alpha-ketoacids has been confirmed both in vitro in several cell types including neurons and in vivo in whole organs such as heart or kidney (Desagher et al., 1997; Das, 2006). Accumulation of reactive oxygen species (ROS) is a prominent feature of oxidative stress and by scavenging ROS pyruvate may substantially reduce the toxic consequences of this pathological event.

\section{ANTI-INFLAMMATORY ACTION OF PYRUVATE}

Many studies on different organs provided evidence that pyruvate (ethyl pyruvate) is an effective anti-inflammatory agent (reviewed in Kao and Fink, 2010). They demonstrated that pyruvate treatment down-regulates activation of the pro-inflammatory transcription factor, $\mathrm{NF}-\mathrm{kB}$, as well as the expression of several pro-inflammatory proteins, such as tumor necrosis factor (TNF), interleukin 6 (IL-6) and others (Das, 2006; Kao and Fink, 2010). The mechanism of this pyruvate effect is yet unclear although it may be explained, at least partly, by the pyruvate antioxidant properties as well as by the pyruvate-induced inhibition of poly-ADP ribose polymerase-1 

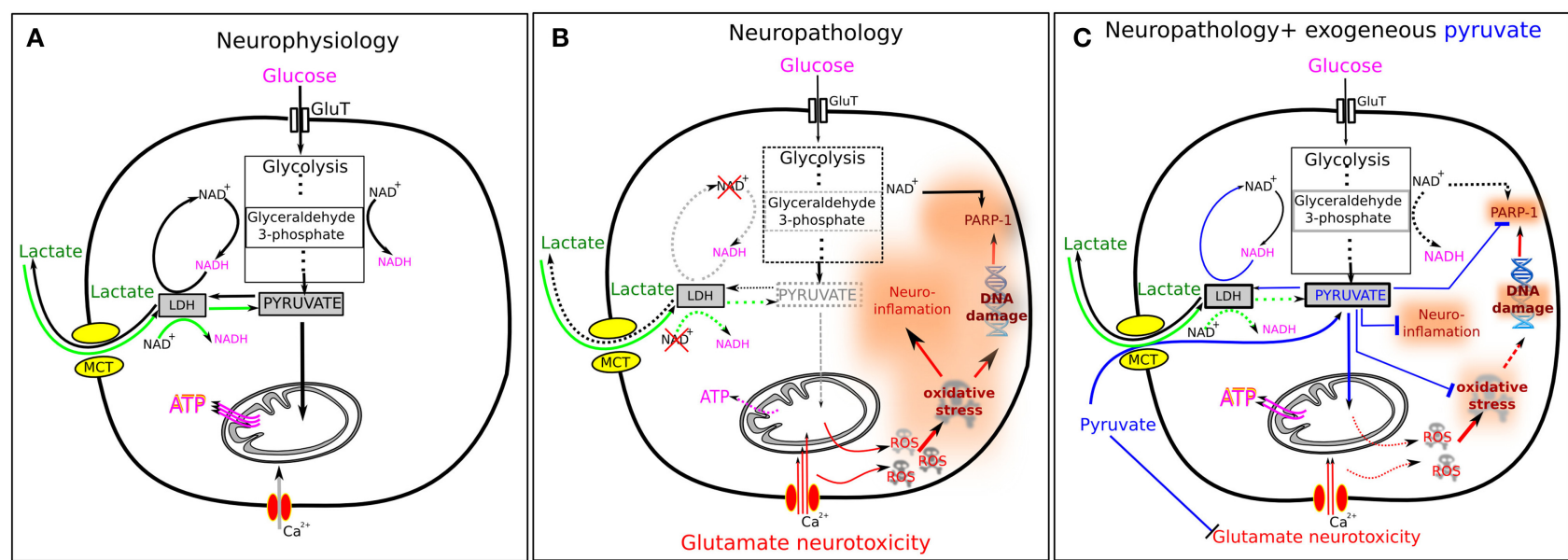

FIGURE 1 | Pyruvate and lactate in normal and pathological conditions. (A) In normal conditions, glucose enters the cell via glucose transporters (GluT) and is metabolized in a 10-step glycolysis. Endogenous pyruvate is a final product of glycolysis and the main energy substrate for ATP generation in mitochondria. Excessive for mitochondria pyruvate can be transformed to lactate by lactate dehydrogenase $(\mathrm{LDH})$ in the presence of $\mathrm{NADH}$. Extracellular lactate can enter the cell via monocarboxylate transporters (MCT). Lactate then is converted to pyruvate by LDH in the presence of $\mathrm{NAD}^{+}$(green arrows). This explains why extracellular lactate may serve efficiently as the energy fuel for brain cells. (B) The situation is changed radically under pathological conditions. A number of neurological disorders are characterized by the oxidative stress and increased level of interstitial glutamate-both factors inducing strong excitotoxicity. Oxidative stress results from excessive presence of ROS, while glutamate induces cellular overload with $\mathrm{Ca}^{2+}$ ions. ROS induce DNA damage leading to the overactivation of poly-ADP ribose polymerase-1 (PARP-1) that results in depletion of cytosolic $\mathrm{NAD}^{+}$. ROS also activate the pro-inflammatory transcription factor NF-kB inducing neuroinflammation. Depletion of $\mathrm{NAD}^{+}$ induces inhibition of glycolysis since the glycolysis step 6, conversion of glyceraldehyde 3-phosphate to 3-phosphoglycerate, requires two molecules of $\mathrm{NAD}^{+}$. This results in the insufficient outcome of pyruvate and decline in the mitochondrial ATP production. Moreover, NAD ${ }^{+}$depletion makes ineffectual the conversion of lactate to pyruvate (dashed green arrows) and lactate cannot serve as the energy substrate anymore. (C) Exogenous pyruvate is able to ameliorate many impaired cellular functions described in (B). In blood, pyruvate activates a blood resident enzyme glutamate-pyruvate transaminase which transforms glutamate into 2-ketoglutarate and thus lowers the blood glutamate level. This results in an enhanced efflux of glutamate from brain parenchyma that reduces neuronal overload with $\mathrm{Ca}^{2+}$ ions. Pyruvate reacts directly with $\mathrm{H}_{2} \mathrm{O}_{2}$ producing acetate, $\mathrm{H}_{2} \mathrm{O}$ and $\mathrm{CO}_{2}$ and thus reducing oxidative stress. Pyruvate inhibits PARP-1 overactivation that prevents depletion of $\mathrm{NAD}^{+}$and thus promotes glycolysis

(glyceraldehyde-3-phosphate depending step). It also inhibits expression of several pro-inflammatory proteins, such as tumor necrosis factor (TNF), interleukin 6 (IL-6) and others. All these effects explain the neuroprotective properties of pyruvate.
(PARP-1) overactivation (see below and Figure 1C).

\section{PYRUVATE ENHANCES GLYCOGEN CONTENT IN ASTROCYTES}

Pyruvate supplementation prior to glucose deprivation significantly protected synaptic function against the deleterious effects of hypoglycemia in brain slices (Shetty et al., 2012). The authors associated beneficial effect of pyruvate with both increased glycogen content during pyruvate pretreatment and subsequent glycogen utilization during glucose deprivation leading to the increased ATP levels. Interestingly, both extra glucose and lactate pretreatment also increased the glycogen content, although significantly less efficiently than pyruvate. However, neither lactate nor extra glucose pretreatment was sufficient to provide the protective effect on synaptic transmission during glucose deprivation.
Pyruvate chronic supplementation also strongly increased the glycogen content of cortical tissue in vivo in the Alzheimer's disease mouse model (APPswe/PS1dE9) (Zilberter et al., 2013).

\section{PYRUVATE PROVIDES NEUROPROTECTION AGAINST DAMAGE INDUCED BY POLY-ADP RIBOSE POLYMERASE-1 OVERACTIVATION}

Poly-ADP ribose polymerase 1 (PARP-1) synthesizes polymers of ADP-ribose that are implicated in regulation of a number of cellular processes including modulation of transcription, DNA repair, neuronal survival and death (Smith et al., 2013). Importantly, to generate polymers of ADP-ribose PARP-1 consumes cytoplasmic $\mathrm{NAD}^{+}$. In various neurological disorders, excessive activation of PARP1 by oxidative stress has been documented (Ma et al., 2012). This process compromised cell survival via activation of pro-death pathways by ADP-ribose polymers and by creating energy deficit via depletion of cytoplasmic $\mathrm{NAD}^{+}$that was followed by inhibition of glycolysis and ATP production (see Figure 1B).

It has been also reported recently that PARP-1 directly inhibits hexokinase (Andrabi et al., 2014), increasing its potential for blocking glycolysis. Importantly, Ying and colleagues reported (Ying et al., 2002) that exogenous TCA cycle substrates (including pyruvate) administration following PARP-1 activation reduced cell death in the astrocyteneuron cultures from approximately $70 \%$ to $30 \%$.

Similar neuroprotective effects of pyruvate was reported in vivo in transient cerebral ischemia and severe hypoglycemia models, in which PARP-1 had been shown to be a key mediator of neurotoxicity (Suh et al., 2003; Moroni and Chiarugi, 
2009). In these models, pyruvate treatment either completely prevented the neuronal loss or reduced it by $70-90 \%$ (Lee et al., 2001; Suh et al., 2005). Brain damage reduction due to pyruvate treatment was also reported in the rodent model of traumatic brain injury with documented prominent oxidative stress, PARP-1 overactivation and loss of $\mathrm{NAD}^{+}$(Satchell et al., 2003; Clark et al., 2007; Fukushima et al., 2009; Sharma et al., 2009). Venous infusion of pyruvate after controlled arterial hemorrhage in swine reduced oxidative stress and PARP fragmentation in the brain (Mongan et al., 2003). Although elucidating the exact mechanisms of pyruvate neuroprotection was beyond the scope of these studies, the authors suggested that the pyruvate action includes the ROS scavenging, $\mathrm{NAD}^{+}$replenishment, recovering the pyruvate-dehydrogenase activity and direct mitochondrial fueling.

Interestingly, PARP-1 overactivation was also demonstrated in the brain of transgenic Alzheimer's disease mouse model (Abeti et al., 2011). In mixed cultures of neurons and glial cells, $\beta$-amyloid peptide, the major neurotoxic agent in the pathophysiology of Alzheimer's disease, evokes oxidative stress followed by hyperactivation of PARP-1, depolarization of mitochondrial membrane and finally cell death. (Abeti and Duchen, 2012). Addition of pyruvate to culture medium of $\beta$-amyloid treated cells prevented the mitochondrial membrane potential loss (Abramov and Duchen, 2005) and improved cell survival (Alvarez et al., 2003).

One reasonable explanation for the efficient pyruvate action may be in its antioxidant properties. Since PARP-1 is activated in response to oxidative damage to DNA, reducing oxidative stress would decrease PARP-1 activity resulting in $\mathrm{NAD}^{+}$depletion. In addition, exogenous pyruvate can provide energy in conditions when glycolysis intensity is reduced due to a low cytoplasmic $\mathrm{NAD}^{+}$. Indeed, pyruvate is a "direct" energy substrate for mitochondria, while lactate needs to be converted first to pyruvate in the reaction dependent on the availability of cytoplasmic $\mathrm{NAD}^{+}$. Importantly, mitochondrial pool of $\mathrm{NAD}^{+}$, indispensible for pyruvate metabolism in mitochondria, is maintained for at least $24 \mathrm{~h}$ when cytoplasmic $\mathrm{NAD}^{+}$is depleted (Stein and Imai, 2012), thus ensuring energy production.

\section{ANTIEPILEPTIC EFFECTS OF PYRUVATE}

Recently, a robust antiepileptic effect of pyruvate (combined with antioxidants ascorbic acid and alpha-tocopherol) treatment has been revealed in the genetic model of temporal lobe epilepsy (Simeone et al., 2014). In addition, the authors showed that a single pretreatment of wild-type mice with these drugs reduced the severity of kainate-induced events resulting in $100 \%$ protection from severe tonic-clonic seizures. Unfortunately, the authors did not determine the contribution of each applied drug to the antiepileptic effect. To the best of our knowledge neither ascorbic acid nor alpha-tocopherol expresses significant antiepileptic action (Waldbaum and Patel, 2010). Therefore, we believe that pyruvate is the major player in the Simeone's work and the pyruvate antiepeileptic effect is presumably reinforced by complementary antioxidants.

Neuronal hyperactivity leading to abnormal oscillations and epilepsy, characteristic for Alzheimer's disease (Amatniek et al., 2006; Noebels, 2011), has been observed as well in different mouse models of the disease (Palop and Mucke, 2009). We found (Minkeviciene et al., 2009; Zilberter et al., 2013) that the general reason for hyperactivity may be the $A \beta$-induced modification of basic neuronal properties, such as the resting membrane potential and reversal potential of GABA-induced currents, presumably evoked by energy metabolism imbalance. Critically, in the presence of pyruvate, $\mathrm{A} \beta$ failed to induce its deleterious effects on the cellular parameters. Moreover, pyruvate chronic dietary supplementation considerably reduced epileptic phenotype in APP/PS1 mice (Zilberter et al., 2013). In another Alzheimer's disease model (3xTg-AD mice), chronic pyruvate treatment reduced both oxidative stress and hyperexcitability, and inhibited short and long-term memory deficits (Isopi et al., 2014).

\section{CONCLUSIONS}

Oxidative stress and metabolic dysfunction are significant pathogenic factors contributing to neurological disorders. Pyruvate may be a unique therapeutic tool for correcting neuronal network abnormalities developing due to these factors. Combination of the following properties validates this conclusion: (i) Oxidative stress is the general feature of neurological disorders and is associated with accumulation of ROS. Pyruvate is a potent scavenger of ROS and its contribution to the antioxidant defense system becomes significant during neuropathologies; (ii) The oxidative stress-induced overactivation of PARP-1 results in the depletion of cytosolic $\mathrm{NAD}^{+}$and inhibition of glycolysis that evokes energy deficiency and frequently results in a cell death. Pyruvate significantly abates overactivation of PARP-1. In addition, as pyruvate is a direct substrate for mitochondrial metabolism and its oxidation does not depend on the cytoplasmic redox state, pyruvate bypasses restrictions imposed by PARP-1 and can restore energy deficiency in such conditions; (iii) Pyruvate reduces the blood glutamate level, facilitating the glutamate efflux from brain tissue through the blood-brain barrier thus reducing the glutamateinduced neurotoxicity; (iv) Pyruvate augements glycogen stores, thus increasing neuronal tolerance to ischemia and hypoglycemia; (v) Neuroinflammation is a common attribute of a number of neuropathologies. Pyruvate reveals a potent anti-inflammatory action; (vi) Pyruvate prevents neural network hyperexcitability.

We conclude that pyruvate, in addition to its well-recognized function in energy metabolism, is a powerful neuroprotector, the potential therapeutic significance of which is yet widely underrated.

\section{ACKNOWLEDGMENTS}

We thank Drs. Tanya Zilberter and Misha Zilberter for their help in manuscript preparation. Our work was supported by the Alzheimer's Association research grant NESAD-12-242486.

\section{REFERENCES}

Abeti, R., Abramov, A. Y., and Duchen, M. R. (2011). Beta-amyloid activates PARP causing astrocytic metabolic failure and neuronal death. Brain 134, 1658-1672. doi: 10.1093/brain/awr104

Abeti, R., and Duchen, M. R. (2012). Activation of PARP by oxidative stress induced by beta-amyloid: implications for Alzheimer's disease. Neurochem. 
Res. 37, 2589-2596. doi: 10.1007/s11064-0120895-x

Abramov, A. Y., and Duchen, M. R. (2005). The role of an astrocytic NADPH oxidase in the neurotoxicity of amyloid beta peptides. Philos. Trans. R. Soc. Lond. B Biol. Sci. 360, 2309-2314. doi: 10.1098/rstb.2005.1766

Alvarez, G., Ramos, M., Ruiz, F., Satrustegui, J., and Bogonez, E. (2003). Pyruvate protection against beta-amyloid-induced neuronal death: role of mitochondrial redox state. J. Neurosci. Res. 73, 260-269. doi: 10.1002/jnr.10648

Amatniek, J. C., Hauser, W. A., Delcastillo-Castaneda, C., Jacobs, D. M., Marder, K., Bell, K., et al. (2006). Incidence and predictors of seizures in patients with Alzheimer's disease. Epilepsia 47, 867-872. doi: 10.1111/j.1528-1167.2006. 00554.x

Andrabi, S. A., Umanah, G. K., Chang, C., Stevens, D. A., Karuppagounder, S. S., Gagne, J. P., et al. (2014). Poly(ADP-ribose) polymerasedependent energy depletion occurs through inhibition of glycolysis. Proc. Natl. Acad. Sci. U.S.A. 111, 10209-10214. doi: 10.1073/pnas.14051 58111

Cai, H., Cong, W. N., Ji, S., Rothman, S., Maudsley, S., and Martin, B. (2012). Metabolic dysfunction in Alzheimer's disease and related neurodegenerative disorders. Curr. Alzheimer Res. 9, 5-17. doi: 10.2174/156720512799015064

Carvalho, A. S., Torres, L. B., Persike, D. S., Fernandes, M. J., Amado, D., Naffah-Mazzacoratti Mda, G., et al. (2011). Neuroprotective effect of pyruvate and oxaloacetate during pilocarpine induced status epilepticus in rats. Neurochem. Int. 58, 385-390. doi: 10.1016/j.neuint.2010. 12.014

Clark, R. S., Vagni, V. A., Nathaniel, P. D., Jenkins, L. W., Dixon, C. E., and Szabo, C. (2007). Local administration of the poly(ADP-ribose) polymerase inhibitor INO-1001 prevents NAD+ depletion and improves water maze performance after traumatic brain injury in mice. J. Neurotrauma 24 , 1399-1405. doi: 10.1089/neu.2007.0305

Das, U. N. (2006). Is pyruvate an endogenous antiinflammatory molecule? Nutrition 22, 965-972. doi: 10.1016/j.nut.2006.05.009

Desagher, S., Glowinski, J., and Premont, J. (1997). Pyruvate protects neurons against hydrogen peroxide-induced toxicity. J. Neurosci. 17, 9060-9067.

Fukushima, M., Lee, S. M., Moro, N., Hovda, D. A., and Sutton, R. L. (2009). Metabolic and histologic effects of sodium pyruvate treatment in the rat after cortical contusion injury. J. Neurotrauma 26, 1095-1110. doi: 10.1089/neu.2008.0771

Gottlieb, M., Wang, Y., and Teichberg, V. I. (2003). Blood-mediated scavenging of cerebrospinal fluid glutamate. J. Neurochem. 87, 119-126. doi: 10.1046/j.1471-4159.2003.01972.x

Heneka, M. T., Kummer, M. P., and Latz, E. (2014). Innate immune activation in neurodegenerative disease. Nat. Rev. Immunol. 14, 463-477. doi: 10.1038/nri3705

Holleman, A. F. (1904). Notice sur l'action de l'eau oxygénée sur les acides $\alpha$-cétoniques et sur les dicétones 1. 2. Recueil des Travaux Chimiques des Pays-Bas et de la Belgique 23, 169-172. doi: 10.1002/recl.19040230504
Isopi, E., Granzotto, A., Corona, C., Bomba, M., Ciavardelli, D., Curcio, M., et al. (2014). Pyruvate prevents the development of age-dependent cognitive deficits in a mouse model of Alzheimer's disease without reducing amyloid and tau pathology. Neurobiol. Dis. doi: 10.1016/j.nbd.2014. 11.013. [Epub ahead of print].

Kao, K. K., and Fink, M. P. (2010). The biochemical basis for the anti-inflammatory and cytoprotective actions of ethyl pyruvate and related compounds. Biochem. Pharmacol. 80, 151-159. doi: 10.1016/j.bcp.2010.03.007

Lee, J. Y., Kim, Y. H., and Koh, J. Y. (2001). Protection by pyruvate against transient forebrain ischemia in rats. J. Neurosci. 21:RC171.

Ma, Y., Chen, H., He, X., Nie, H., Hong, Y., Sheng, C., et al. (2012). NAD+ metabolism and $\mathrm{NAD}(+)$-dependent enzymes: promising therapeutic targets for neurological diseases. Curr. Drug Targets 13, 222-229. doi: 10.2174/138945012799 201711

Melo, A., Monteiro, L., Lima, R. M., Oliveira, D. M., Cerqueira, M. D., and El-Bacha, R. S. (2011). Oxidative stress in neurodegenerative diseases: mechanisms and therapeutic perspectives. Oxid. Med. Cell. Longev. 2011:467180. doi: $10.1155 / 2011 / 467180$

Minkeviciene, R., Rheims, S., Dobszay, M. B., Zilberter, M., Hartikainen, J., Fülöp, L., et al. (2009). Fibrillar $\beta$-amyloidinduced hyperexcitability of cortical and hippocampal neurons triggers progressive epilepsy. J. Neurosci. 29, 3453-3462. doi: 10.1523/JNEUROSCI.5215-08.2009

Mongan, P. D., Karaian, J., Van Der Schuur, B. M., Via, D. K., and Sharma, P. (2003). Pyruvate prevents poly-ADP ribose polymerase (PARP) activation, oxidative damage, and pyruvate dehydrogenase deactivation during hemorrhagic shock in swine. J. Surg. Res. 112, 180-188. doi: 10.1016/S00224804(03)00148-3

Moroni, F., and Chiarugi, A. (2009). Post-ischemic brain damage: targeting PARP-1 within the ischemic neurovascular units as a realistic avenue to stroke treatment. FEBS J. 276, 36-45. doi: 10.1111/j.1742-4658.2008.06768.x

Noebels, J. (2011). A perfect storm: converging paths of epilepsy and Alzheimer's dementia intersect in the hippocampal formation. Epilepsia (Suppl. 1), 39-46. doi: 10.1111/j.1528-1167.2010. 02909.x

Palop, J. J., and Mucke, L. (2009). Epilepsy and cognitive impairments in Alzheimer disease. Arch. Neurol. 66, 435-440. doi: 10.1001/archneurol.2009.15

Satchell, M. A., Zhang, X., Kochanek, P. M., Dixon, C. E., Jenkins, L. W., Melick, J., et al. (2003). A dual role for poly-ADP-ribosylation in spatial memory acquisition after traumatic brain injury in mice involving NAD+ depletion and ribosylation of 14-3-3gamma. J. Neurochem. 85, 697-708. doi: 10.1046/j.1471-4159.2003. 01707.x

Sharma, P., Benford, B., Li, Z. Z., and Ling, G. S. (2009). Role of pyruvate dehydrogenase complex in traumatic brain injury and Measurement of pyruvate dehydrogenase enzyme by dipstick test. J. Emerg. Trauma Shock 2, 67-72. doi: $10.4103 / 0974-2700.50739$
Shetty, P. K., Sadgrove, M. P., Galeffi, F., and Turner, D. A. (2012). Pyruvate incubation enhances glycogen stores and sustains neuronal function during subsequent glucose deprivation. Neurobiol. Dis. 45, 177-187. doi: 10.1016/j.nbd.2011.08.002

Simeone, K. A., Matthews, S. A., Samson, K. K., and Simeone, T. A. (2014). Targeting deficiencies in mitochondrial respiratory complex I and functional uncoupling exerts anti-seizure effects in a genetic model of temporal lobe epilepsy and in a model of acute temporal lobe seizures. Exp. Neurol. 251, 84-90. doi: 10.1016/j.expneurol.2013.11.005

Smith, J. A., Park, S., Krause, J. S., and Banik, N. L. (2013). Oxidative stress, DNA damage, and the telomeric complex as therapeutic targets in acute neurodegeneration. Neurochem. Int. 62, 764-775. doi: 10.1016/j.neuint.2013.02.013

Stein, L. R., and Imai, S. (2012). The dynamic regulation of NAD metabolism in mitochondria. Trends Endocrinol. Metab. 23, 420-428. doi: 10.1016/j.tem.2012.06.005

Suh, S. W., Aoyama, K., Chen, Y., Garnier, P., Matsumori, Y., Gum, E., et al. (2003). Hypoglycemic neuronal death and cognitive impairment are prevented by poly(ADPribose) polymerase inhibitors administered after hypoglycemia. J. Neurosci. 23, 10681-10690.

Suh, S. W., Aoyama, K., Matsumori, Y., Liu, J., and Swanson, R. A. (2005). Pyruvate administered after severe hypoglycemia reduces neuronal death and cognitive impairment. Diabetes 54, 1452-1458. doi: 10.2337/diabetes.54.5.1452

Teichberg, V. I., Cohen-Kashi-Malina, K., Cooper I., and Zlotnik, A. (2009). Homeostasis of glutamate in brain fluids: an accelerated brain-to-blood efflux of excess glutamate is produced by blood glutamate scavenging and offers protection from neuropathologies. Neuroscience 158, 301-308. doi: 10.1016/j.neuroscience.2008.02.075

Waldbaum, S., and Patel, M. (2010). Mitochondria, oxidative stress, and temporal lobe epilepsy. Epilepsy Res. 88, 23-45. doi: 10.1016/j.eplepsyres.2009.09.020

Wang, Y., and Qin, Z. H. (2010). Molecular and cellular mechanisms of excitotoxic neuronal death Apoptosis 15, 1382-1402. doi: 10.1007/s10495-0100481-0

Ying, W., Chen, Y., Alano, C. C., and Swanson, R. A. (2002). Tricarboxylic acid cycle substrates prevent PARP-mediated death of neurons and astrocytes. J. Cereb. Blood Flow Metab. 22, 774-779. doi 10.1097/00004647-200207000-00002

Zilberter, M., Ivanov, A., Ziyatdinova, S., Mukhtarov, M., Malkov, A., Alpar, A., et al. (2013). Dietary energy substrates reverse early neuronal hyperactivity in a mouse model of Alzheimer's disease. J. Neurochem. 125, 157-171. doi: 10.1111/jnc. 12127

Zlotnik, A., Gurevich, B., Cherniavsky, E., Tkachov, S., Matuzani-Ruban, A., Leon, A., et al. (2008). The contribution of the blood glutamate scavenging activity of pyruvate to its neuroprotective properties in a rat model of closed head injury. Neurochem. Res. 33, 1044-1050. doi: 10.1007/s11064-007-9548-x

Zlotnik, A., Sinelnikov, I., Gruenbaum, B. F., Gruenbaum, S. E., Dubilet, M., Dubilet, E., et al. (2012). Effect of glutamate and blood glutamate scavengers oxaloacetate and pyruvate 
on neurological outcome and pathohistology of the hippocampus after traumatic brain injury in rats. Anesthesiology 116, 73-83. doi: 10.1097/ALN.0b013e31823d7731

Conflict of Interest Statement: The authors declare that the research was conducted in the absence of any commercial or financial relationships that could be construed as a potential conflict of interest.
Received: 27 October 2014; accepted: 12 January 2015; published online: 17 February 2015.

Citation: Zilberter Y, Gubkina $O$ and Ivanov AI (2015) A unique array of neuroprotective effects of pyruvate in neuropathology. Front. Neurosci. 9:17. doi: 10.3389/ fnins.2015.00017

This article was submitted to Neuroenergetics, Nutrition and Brain Health, a section of the journal Frontiers in Neuroscience.
Copyright (c) 2015 Zilberter, Gubkina and Ivanov. This is an open-access article distributed under the terms of the Creative Commons Attribution License (CC BY). The use, distribution or reproduction in other forums is permitted, provided the original author(s) or licensor are credited and that the original publication in this journal is cited, in accordance with accepted academic practice. No use, distribution or reproduction is permitted which does not comply with these terms. 